\title{
Phenomenological model of nonequilibrium solidification
}

\author{
L.M. Martyushev*, A.S. Soboleva \\ Ural Federal University, 19 Mira Str., Ekaterinburg, 620002, Russia \\ Institute of Industrial Ecology, 20 S. Kovalevskaya Str., Ekaterinburg, 620219, Russia
}

\section{H I G H L I G H T S}

- The maximum entropy production principle is considered as a foundation of nonequilibrium pattern formation.

- Based on the principle, a simple phenomenological model of dendrite growth is developed.

- The model results quantitatively agree with the experimental data.

\section{A R T I C L E I N F O}

\section{Article history:}

Received 3 May 2013

Received in revised form 25 June 2013

Available online 25 July 2013

\section{Keywords:}

Maximum entropy production principle

Dendrite growth

\begin{abstract}
A B S T R A C T
The maximum entropy production principle is used as a foundation for the nonequilibrium solidification theory. Based on this principle, a new simple model of dendrite solidification is proposed. The model predicts the explicit dependency of a dendrite's rate and tip size on supercooling. The obtained results are devoid of the contradictions of the previous models and show quantitative agreement with the recent experimental data for the SCN dendrite.
\end{abstract}

(c) 2013 Elsevier B.V. All rights reserved.

\section{Introduction}

Nonequilibrium growth of crystals from different media is typical for many natural and technological processes. Such growth results in the complex, intricate shape of crystals. In terms of appearance, they are divided into dendrites, nonsymmetrical diffusion-limited aggregates (DLA), seaweed-like structures, etc. [1-7]. It is interesting to note here that similar shapes are typical not only for crystal systems but for some biotic systems (e.g. for bacterial colonies) in the process of selforganization (see e.g., Ref. [3]). Currently, most investigations (both theoretical and experimental) deal with the dendrite growth for which a snowflake forming in air supersaturated with water vapor is a typical example. One of the core issues that have been discussed for more than fifty years is the connection between three quantities characterizing the dendrite (see, for example, the reviews [8-13]). These quantities are the dendrite growth rate $v$, the dendrite tip size, which is conventionally described by the curvature radius $\rho$, and the degree of nonequilibrium of the system $\Delta$ (a relative quantity of supercooling or supersaturation in the case of crystallization from the melt or from the solution/vapor, respectively). According to the experiments, in the case of preset $\Delta$, the dendrite has certain values of $v$ and $\rho$ during its growth. The microscope solvability theory ${ }^{1}$ is presently the most common method for determining the dependencies $v(\Delta)$ and $\rho(\Delta)$ [11-14]. This theory analytically studies the growth and stability of a needle crystal by means of the rigorous solution of a heat-conduction problem with the anisotropies of surface energy and kinetic effects. This theory allows predicting the behavior of $v(\Delta)$ and $\rho(\Delta)$ for a number of the simplest cases of dendrite growth. However, such a method cannot be considered as a basis for material and metallurgical applications because there are no explicit dependencies (and fitting formulas) $v(\Delta)$ and $\rho(\Delta)$ convenient for

\footnotetext{
* Corresponding author at: Ural Federal University, 19 Mira Str., Ekaterinburg, 620002, Russia.

E-mail addresses: LeonidMartyushev@gmail.com, mlm@ecko.uran.ru (L.M. Martyushev).

1 Further referred to as MST for brevity.
} 
practical use ${ }^{2}$ [13]. At the same time, there is another drawback, in our opinion, the most critical. The dendrite crystallization theory has appeared and been developed as some special problem of mathematical physics ${ }^{3}$ intended only for describing dendrite growth. ${ }^{4}$ As a result, in a number of cases the selected mathematical method of problem analysis can lead to principally different physical conclusions (for example, in regard to the prerequisites for existence of the stable dendrite tip or to the mechanism of sidebranch appearance (see Refs. $[13,14,16,17])$ ). The development of simple phenomenological models based on a minimum number of statements, preferably the most fundamental ones, ${ }^{5}$ can be a possible way out from the currently observed stagnation in the dendrite growth theory. The model shall be constructed in general for nonequilibrium solidification (because the dendrite is only one of many nonequilibrium shapes of growth; and according to the experiment, these shapes can be simultaneously observed in the course of nonequilibrium growth $[18,19])$. The development of such a phenomenological model is the objective hereof.

\section{Model}

The maximum entropy production principle, which is currently considered as one of the most important principles of nonequilibrium physics (see reviews [20-22]) finding increased usage in the problems of nonequilibrium crystallization [22-27], is chosen as a foundation. This principle can be most generally formulated as follows: at each level of description, with preset external constraints, the relationship between the cause and the response of a nonequilibrium system is established such as to maximize the entropy production. The maximum entropy production principle allows determining the dependency of thermodynamic fluxes on forces through maximization of the local entropy production ${ }^{6}$ under the given thermodynamic forces. As a result, the principle leads to the equations describing heat/mass transfer (both molecular and convectional) which are traditionally used for the mathematical treatment of dendrite crystallization.

For the simplest one-component system solidifying from the supercooling melt, it is known (see e.g., Ref. [25]) that the local entropy production is proportional to the squared local rate of crystal growth $V$. Obviously, the supercooling $\Delta$ is the thermodynamic force here. For this simplest case, the maximization of the local entropy production agrees with the maximization $V$ under the given (fixed) supercooling $\Delta$ :

$$
V \rightarrow \max \text {. }
$$

Thus, the condition (1) constitutes the foundation of the present model of nonequilibrium growth of a crystal (the dendrite, in the particular case). In anticipation of multiple criticisms regarding the statement (1), let us make a number of important notes explaining our viewpoint.

(1) The maximization of growing dendrite rate (1) was repeatedly used in the past, especially in the middle of the twentieth century $[8,8,12,16,22,23,25]$. Such an approach provides a quadratic dependency of rate on supercooling. However, this method was subsequently abandoned because the obtained results appeared to be in poor agreement with experiment: the growth rate was too high and the tip radius was too small. It is conventional to attribute the poor agreement with experiment specifically to the rate maximization procedure (1). However, it seems that the true reason may lie in the fact that the used models to which the maximization was applied misrepresented the real phenomena under consideration. Specifically, in the case of dendrite growth from the melt under terrestrial conditions, the convective heat transfer was obviously underestimated, which influence, according to the modern thorough experiments, is significant [13].

(2) As is known, MST is presently a common approach to describe the dendrite tip. This theory originated approximately thirty years after the disappointment in, and rejection of, the rate maximization principle. It is paradoxical that, in the course of the evolution of the theory, the founders of MST came to the conclusion that the solution describing dendrite growth at the maximum possible rate is the only linearly stable solution out of the discrete spectra of stationary "needle-shaped" solutions [11]. Thus, it turned out to be that the statement of rate maximality follows from MST! Here, it is appropriate to also note that some researchers (for example, E. Ben-Jacob [2]) seeing a number of problems in MST itself (specifically, in explaining the results of the anisotropic Hele-Shaw experiment) made the following statement: if more than one morphology is a possible solution, only the fastest growing morphology is nonlinearly stable and will be observed. So, as a result of the long-term intensive development of the dendrite growth theory, the researchers came back to the maximum growth rate principle.

(3) Whereas more than half a century ago the maximum rate principle appeared by intuition based on common sense, today it directly follows from the first principles of nonequilibrium thermodynamics (the maximum entropy production principle). That is an advantage of such an approach as compared to a number of modern theories of dendrite growth lacking any sound foundation.

\footnotetext{
2 There are only numerical and/or asymptotic dependencies.

3 Here, the field of study based on and developed in the paper by Ivantsov [15] is meant. These studies represent the overwhelming majority and serve as a basis for multiple modern reviews, monographs, and textbooks on dendrite growth [11-13].

4 Initially, a very particular model was chosen: an isothermal paraboloid growing at the constant rate in the medium due to heat conduction only. However, even this model itself had no analytical solution. Then, it has been "improved" and generalized for decades, which resulted in the avalanche-like growth of its mathematical complexity.

5 Subsequently, if required, the model can be supplemented with assumptions taking into account the specifics of this or that nonequilibrium growth.

6 In the general case, this dependency may be nonlinear.
} 
The condition (1) needs to be supplemented with some crystal growth model. In this case, let us choose the simplest yet quite common local model representing the heat balance on the crystal surface [28]:

$$
\begin{aligned}
& A \frac{T_{\text {int }}-T_{\infty}}{R}=B\left(T_{s}-T_{\text {int }}\right), \\
& V=B\left(T_{s}-T_{\text {int }}\right),
\end{aligned}
$$

where $R$ is the typical crystal size, $T_{\infty}$ is the temperature at a distance from the growing crystal (at the boundary layer distance, which usually amounts to several $R$ ), $T_{\text {int }}$ is the melt temperature near the arbitrarily-shaped boundary, $T_{s}$ is the melting temperature of the arbitrarily-surfaced crystal, $A$ is the coefficient determining heat transfer in the melt and depending on the heat conduction coefficient, boundary layer thickness, crystal density, latent crystallization heat, etc., ${ }^{7}$ and $B$ is the coefficient determining heat transfer at the crystal boundary and, in the general case, depending on the presence of adsorbed impurities, chemical nature of the crystal at hand, surface temperature, etc. ${ }^{8}$

Let us use a conventional representation for the temperature $T_{s}$ as $[25,28]$ :

$$
T_{s}=T_{0}\left(1-\frac{C}{R}\right),
$$

$T_{0}$ is the equilibrium melting temperature of the crystal's planar boundary (reference quantity), and $C$ is the coefficient determining the deviation of the melting temperature of the planar surface from the one of the curved surface and depending on the surface energy of the crystal-melt boundary, molar volume of the melt, surface curvature, anisotropy, etc.

So, the growth model depends on the three coefficients $A, B$, and $C$, where the first one characterizes heat transfer in the melt volume and the two others characterize properties of the crystal-melt surface. Let us rearrange (2)-(4), by excluding $T_{\text {int }}$, in the form:

$$
V=\frac{B T_{0} \Delta-B C T_{0} / R}{1+B R / A},
$$

where the relative supercooling quantity is introduced $\Delta=\left(T_{0}-T_{\infty}\right) / T_{0}$.

The relation (5) interconnects the three quantities in the case of nonequilibrium crystal growth: growth rate, typical size, and supercooling. This relation is quite general and, together with (1), is valid for multiple cases of nonequilibrium crystallization.

Let us show a number of corollaries of (1), and (5) for the simplest case. Let the coefficients $A, B$, and $C$ be independent from the crystal size and the relative supercooling be invariable (fixed). Thus, the typical size is the only free parameter in (5). According to (1) and (5), the maximum ${ }^{9}$ rate is reached for the typical size of the crystal structure:

$$
R=\frac{B C+\sqrt{B^{2} C^{2}+A B C \Delta}}{B \Delta} .
$$

By inserting the latter in (5), we have:

$$
V=\frac{A B T_{0} \Delta^{2}}{[\sqrt{B C}+\sqrt{B C+A \Delta}]^{2}} .
$$

The obtained explicit expressions (6) and (7) can be used for analytical calculations related to the nonequilibrium crystal growth. However, such dependencies are not always convenient for approximating the experimental data at hand. Let us transform them into approximating polynomials by expanding them into a series in terms of supercooling. Here, it is important to note that the coefficient $A$ can depend on supercooling. Let this dependency have the simplest form ${ }^{10}$ $A=A_{0}+A_{1} \Delta$. Then:

$$
\begin{aligned}
& R=\frac{2 C}{\Delta}+\frac{A_{0}}{2 B}-\frac{A_{0}^{2}-4 A_{1} B C}{8 B^{2} C} \Delta+\cdots \\
& V=\frac{A_{0} T_{0}}{4 C} \Delta^{2}-\frac{T_{0}\left(A_{0}^{2}-2 A_{1} B C\right)}{8 B C^{2}} \Delta^{3}+\cdots
\end{aligned}
$$

\footnotetext{
7 The heat transfer is not restricted only to heat conduction in this expression, it may also be related to convection (in this case, the coefficient $A$ can depend on $R$ and the tip shape).

8 This coefficient is directly related to the so-called kinetic coefficient of crystallization.

9 It can be easily shown that for $A>0, B>0$, and $C>0$ the obtained extreme point corresponds to the maximum.

10 Indeed, on the one hand, the heat conduction coefficient depends on the melt temperature (therefore, also on $\Delta$ ); on the other hand, the assumption of linearity in $\left(T_{s}-T_{\text {int }}\right)$ on the right side of $(2)$ is often too rough [28]. Let us note the importance of the coefficient $A_{1}$ for the described model and the need for its further study.
} 


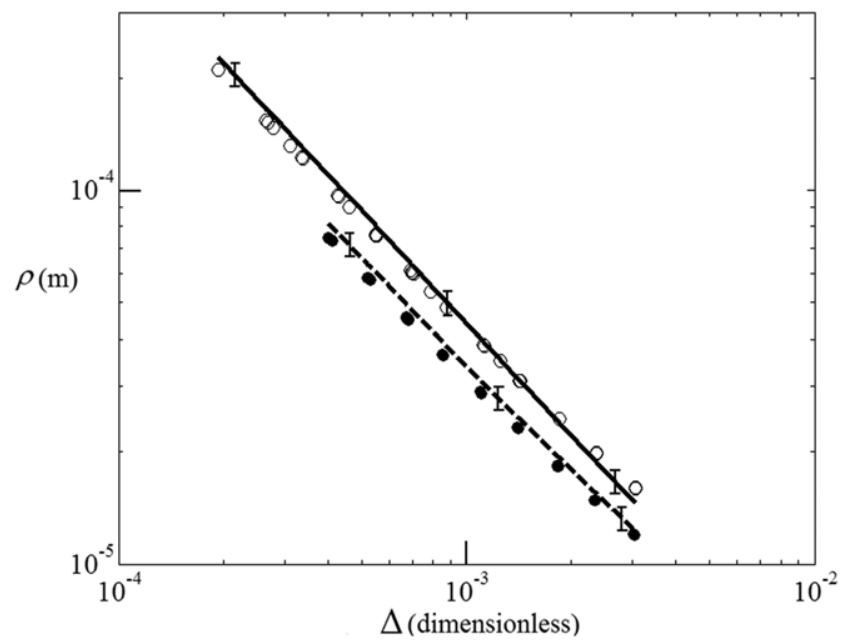

Fig. 1. Dependency of the curvature radius of dendrite tip $\rho$ on the dimensionless supercooling $\Delta$. Experimental data is shown by the circles: empty, for microgravity; filled, for terrestrial conditions. The approximations (10) for microgravity conditions $\left(a=(4.4 \pm 0.4) \cdot 10^{-8}, b=(1.0 \pm 0.1) \cdot 10^{-7}\right)$ and for terrestrial conditions $\left(a=(3.2 \pm 0.3) \cdot 10^{-8}, b=(1.9 \pm 0.2) \cdot 10^{-6}\right)$ are shown by the solid and dashed lines, respectively.

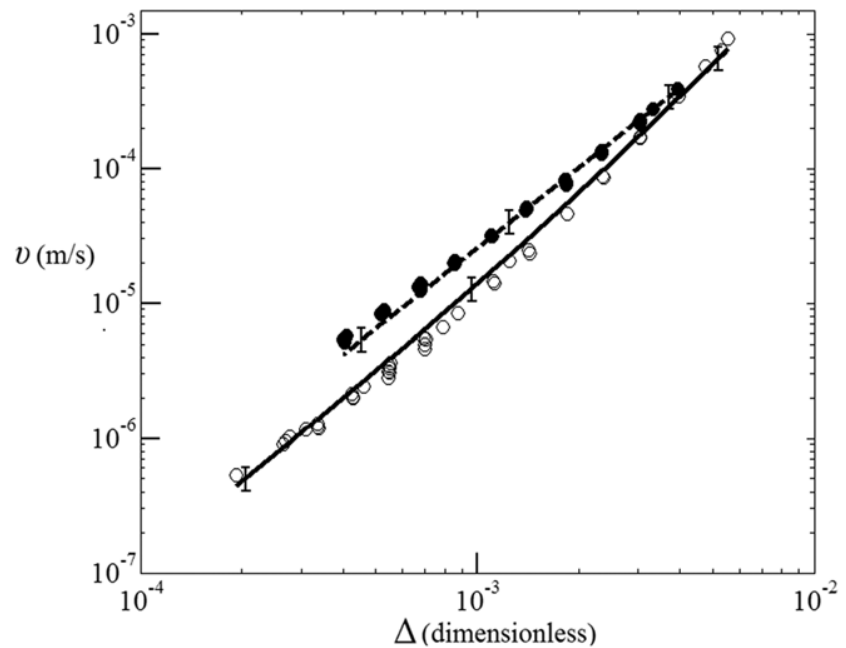

Fig. 2. Dependency of the growth rate of dendrite tip $v$ on the dimensionless supercooling $\Delta$. Experimental data is shown by the circles: empty, for microgravity, filled, for terrestrial conditions. The approximations (11) for microgravity conditions $(c=11.5 \pm 1.2, d=2500 \pm 250)$ and for terrestrial conditions ( $c=26.0 \pm 2.6, d=-300.0 \pm 30.0)$ are shown by the solid and dashed lines, respectively.

\section{Dendrite growth. The model verification}

For verifying our phenomenological model, let us use the experimental data $(v, \rho, \Delta)$ obtained for the dendrite growth of succinonitrile (SCN) under terrestrial and microgravity conditions ${ }^{11}$ [29]. Based on the example under study, let us rename the variables in (8) and (9): $V \rightarrow v$ and $R \rightarrow \rho$; and by limiting ourselves to the first two summands from (8), (9), we obtain:

$$
\begin{aligned}
& \rho=\frac{a}{\Delta}+b, \\
& v=c \Delta^{2}+d \Delta^{3},
\end{aligned}
$$

where, for compactness, the following notation is introduced: $a=2 C, b=A_{0} /(2 B), c=A_{0} T_{0} /(4 C), d=T_{0}\left(2 A_{1} B C-\right.$ $\left.A_{0}^{2}\right) /\left(8 B C^{2}\right)$. Let us note the presence of the free term $(b)$ in $(10)$ independent from supercooling, which is not used in the traditional approximations [12,13]. According to Eqs. (2) and (3), in the simplest case, $b$ is proportional to the ratio of thermal diffusivity to a kinetic coefficient of crystallization. This coefficient is important from the theoretical viewpoint as it allows

\footnotetext{
11 Currently, this data is traditionally used for verifying theories.
} 
a

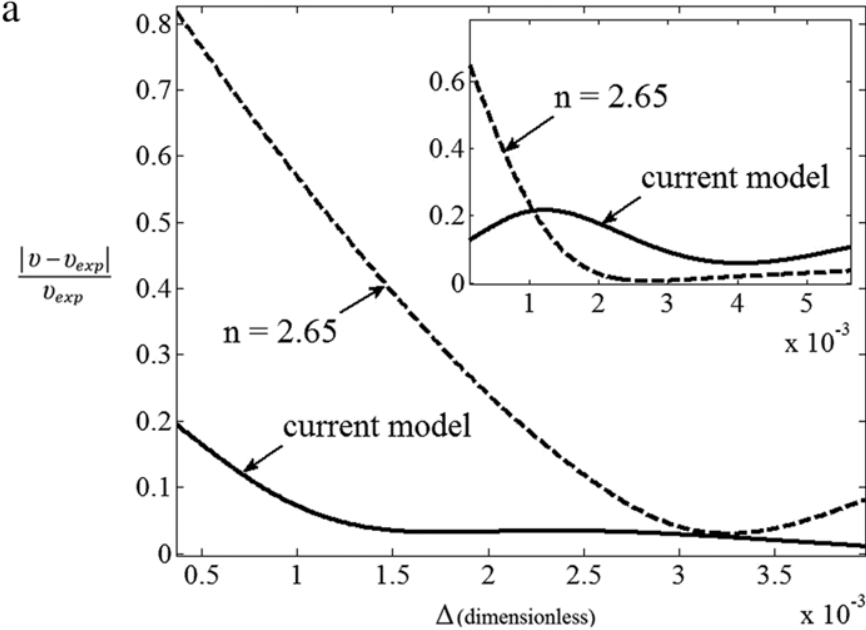

b

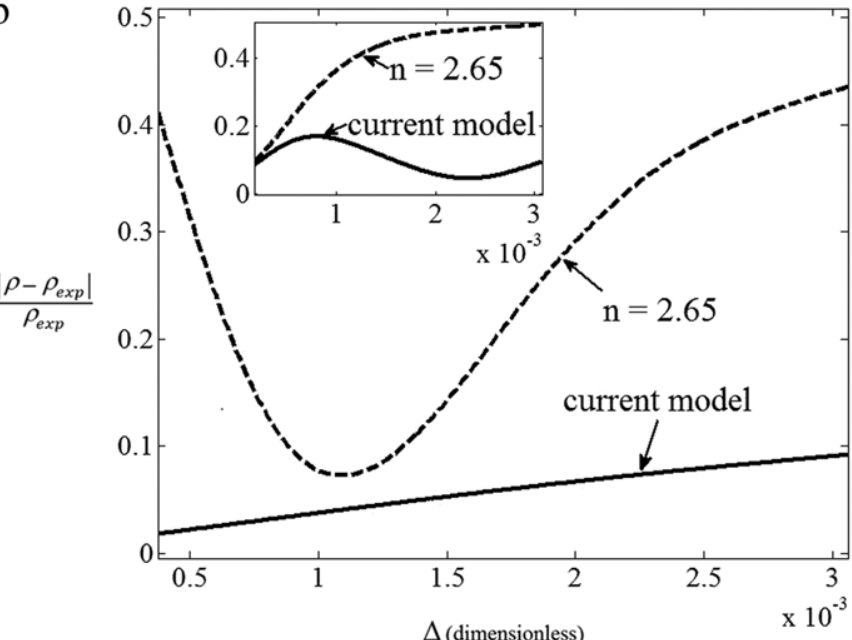

Fig. 3. Dependency of the relative error of approximations of the experimental values of rate $v_{\exp }(\mathrm{a})$ and the curvature radius of dendrite tip $\rho_{\exp }$ (b) on the dimensionless supercooling $\Delta$. The approximations are made using the model (10), and (11), and the model $v \propto \Delta^{n}$ and $\rho \propto \Delta^{-n / 2}(n=2.65$ ). The model coefficients were found using MATLAB software (curve fitting toolbox) taking into account all the available experimental values. The error for terrestrial conditions is shown in the graph and the error for microgravity conditions is shown in the inset.

introducing a lower cut-off value for the limit size of the dendrite tip in the case of relatively high supercoolings. ${ }^{12}$ In the case of such supercoolings, $\rho \rightarrow 0$ and $b / \rho \gg 1$; as a consequence [28], the growth is limited by surface attachment kinetics.

According to Figs. 1 and 2, the approximations (10) and (11) describe the available experimental data for the SCN dendrites over the whole interval of supercooling variation rather well. Let us compare the obtained result with the previously existing one. In the literature, $v \propto \Delta^{n}$ and $\rho \propto \Delta^{-n / 2}(n=2.5-2.65)$ are the most common dependencies. These relations follow from the approximation of numerical solutions of a number of mathematical models of the parabolic-shaped dendrite $^{13}[8,13]$. The relative errors resulting from these approximations and from $(10)$, and (11) proposed herein are given in Fig. 3. It can be seen that the error resulting from (10), and (11) weakly depends on $\Delta$ and does not exceed $20 \%$. In contrast, the dependencies $v \propto \Delta^{n}$ and $\rho \propto \Delta^{-n / 2}$ provide a widely different accuracy for different supercoolings, including above $70 \%$.

The found coefficients of approximation $a, b, c$, and $d$ (see the captions of Figs. 1 and 2) make it possible to determine the basic coefficients of the model (2)-(4): $A_{0}=2 c a / T_{0}, A_{1}=2(d a+2 c b) / T_{0}, B=c a /\left(b T_{0}\right), C=a / 2$. It appears that for the experiment under microgravity conditions $A_{0}=(3.1 \pm 0.6) \cdot 10^{-9}, A_{1}=(6.8 \pm 1.4) \cdot 10^{-7}, B=(1.50 \pm 0.45) \cdot 10^{-2}$, $C=(2.2 \pm 0.2) \cdot 10^{-8}$, whereas under terrestrial conditions $A_{0}=(5.0 \pm 1.0) \cdot 10^{-9}, A_{1}=(5.4 \pm 1.1) \cdot 10^{-7}, B=$ $(0.13 \pm 0.04) \cdot 10^{-2}, C=(1.6 \pm 0.1) \cdot 10^{-8}$. As is seen, the three coefficients $\left(A_{0}, B\right.$, and $\left.C\right)$ reliably differ for the experiments under Earth and microgravity conditions. The high value of $A_{0}$ for the terrestrial conditions is easily explained by the

\footnotetext{
12 Let us note that one of the recent theories of dendrite growth [30] based on dimensional analysis also indicates, in the case of small $\Delta$, the presence of a summand of the $b$ type in the expression for $\rho$.

13 Including MST.
} 
convective contribution; however, the reliable difference for the coefficients $B$ and $C$ requires further analysis. Let us note that the reliable difference in the values of $A_{1}$ was not proved.

Using (10), and (11), we have:

$$
V R^{2}=a^{2} c+a(2 b c+a d) \Delta+\cdots .
$$

Thus, according to the approach developed herein, the quantity $V R^{2}$ depends on supercooling and can be considered as a constant quantity only when $\Delta$ tends to zero. Since $a^{2} c=A_{0} C T_{0}$, this constant quantity is directly proportional to the heat transfer intensity in the melt volume and depends on the properties on the crystal-melt surface. According to the obtained data on the SCN solidification, $a^{2} c$ is equal to $(2.6 \pm 0.9) \cdot 10^{-14}$ under terrestrial conditions and to $(2.2 \pm 0.7) \cdot 10^{-14}$ under microgravity conditions, i.e. these values do not differ reliably. So, in the limit of infinitesimal $\Delta$, the result of the present model can be reduced to the result of MST (according to which this constant is equal to $2.5 \cdot 10^{-14}$ for SNC [29]). With the increase of supercooling, the coefficient responsible for linear growth of $V R^{2}$ proves to be equal to $(2.8 \pm 0.9) \cdot 10^{-12}$ under terrestrial conditions and to $(4.9 \pm 1.6) \cdot 10^{-12}$ under space conditions of crystallization; i.e. these coefficients do not differ reliably. Let us note that these coefficients were calculated from the formula $a(2 b c+a d)$ based on the values of $a, b, c$, and $d$ obtained from the approximation shown in Figs. 1 and 2. If the experimental dependencies of $V R^{2}$ on $\Delta$ are directly linearly approximated, then the results will be as follows: $(3.2 \pm 0.9) \cdot 10^{-12} \Delta+(2.6 \pm 0.1) \cdot 10^{-14}$ for terrestrial conditions of crystallization and $(3.5 \pm 0.7) \cdot 10^{-12} \Delta+(2.5 \pm 0.1) \cdot 10^{-14}$ for crystallization under microgravity conditions. It is important to note that the sum of squares due to error (SSE), in the case of linear approximation, is equal to $1.0 \cdot 10^{-28}$, for microgravity, and to $3.5 \cdot 10^{-28}$, for Earth; whereas in the case of the approximation by a constant quantity it is equal to $6.9 \cdot 10^{-28}$ and $16.3 \cdot 10^{-28}$, respectively, which means that the linear approximation is more preferable.

\section{Conclusion}

Thus, a simple phenomenological model of nonequilibrium crystal growth based on the maximum entropy production principle is proposed herein. It is demonstrated that, as applied to the dendrite growth, the model provides a better quantitative description of the dependency of the growing dendrite's rate and tip size on supercooling. The fundamental differences between the approach proposed herein and the approaches available in the literature (e.g. MST or the one given in Ref. [17]) are as follows. (1) The possibility of both qualitative predication and quantitative description (using the explicit approximation formulas of (10) and (11) types) of a nonequilibrium crystal (not only dendrite) growth under various conditions, for different geometries and over the whole possible interval of supercoolings. Traditional approaches have no such generality and provide solutions (often implicit and inconvenient for practical use) for a very narrow class of problems (on supercooling, on solidification conditions, on geometry, etc.). (2) The maximum entropy production principle is the foundation of the proposed approach. The particular crystallization model performs an important but still an auxiliary function complementing the principle. In the traditional approaches, the statements similar to the mentioned maximum principle either follow from the mathematical solution of a specific problem or originate as auxiliary statements, which allow selecting a unique solution among several solution candidates.

Based on the above, it seems to us that the approach proposed herein can be very useful and fruitful for studying various pattern formations observed under nonequilibrium conditions.

\section{References}

[1] E. Ben-Jacob, Contemp. Phys. 34 (1993) 247.

[2] E. Ben-Jacob, P. Garik, Nature 343 (1990) 523.

[3] E. Ben-Jacob, Philos. Trans. R. Soc. Lond. A 361 (2003) 1283.

[4] T. Ihle, H. Müller-Krumbhaar, Phys. Rev. E 49 (1994) 2972.

[5] H.M. Singer, J.H. Bilgram, Phys. Rev. E 70 (2004) 031601.

[6] H. Honjo, S. Ohta, Y. Sawada, Phys. Rev. Lett. 55 (1985) 841.

[7] Y. Sawada, Physica 140A (1986) 134.

[8] M.E. Glicksman, R.J. Schaefer, J.D. Ayers, Metall. Trans. A 7A (1976) 1747.

[9] J.S. Langer, Rev. Modern Phys. 52 (1980) 1.

[10] D. Kessler, J. Koplik, H. Levine, Adv. Phys. 37 (1988) 255.

[11] E.A. Brener, V.I. Melnikov, Adv. Phys. 40 (1991) 53.

[12] W. Kurz, D.J. Fisher, Fundamentals of solidification, Trans. Tech. Pub. (1992).

[13] M.E. Glicksman, Principles of Solidification. An Introduction to Modern Casting and Crystal Growth Concepts, Springer, 2011.

[14] U. Bisang, J.H. Bilgram, Phys. Rev. E 54 (1996) 5309.

[15] G.P. Ivantsov, Dokl. Akad. Nauk 58 (1947) 567.

[16] M.E. Glicksman, Metall. Mater. Trans. A 43A (2012) 391.

[17] J.J. Xu, D.-S. Yu, J. Cryst. Growth 198/199 (1999) 43.

[18] A.A. Shibkov, Yu.I. Golovin, M.A. Zheltov, et al., Physica A 319 (2003) 65

[19] Y. Sawada, B. Perrin, P. Tabeling, et al., Phys. Rev. A 43 (1991) 5537.

[20] H. Ziegler, An Introduction to Thermomechanics, North-Holland, Amsterdam, 1983.

[21] A. Kleidon, R.D. Lorenz (Eds.), Non-equilibrium Thermodynamics and the Production of Entropy in Life, Earth, and Beyond, Springer-Verlag, Heidelberg, 2004.

[22] L.M. Martyushev, V.D. Seleznev, Phys. Rep. 426 (2006) 1.

[23] J.S. Kirkaldy, Rep. Progr. Phys. 55 (1992) 723

[24] J.A. Sekhar, J. Mater. Sci. 46 (2011) 6172.

[25] L.M. Martyushev, V.D. Seleznev, I.E. Kuznetsova, JETP 91 (2000) 132 
[26] L.M. Martyushev, E.G. Axelrod, JETP Lett. 78 (2003) 476

[27] L.M. Martyushev, E.A. Chervontseva, Phys. Lett. A 373 (2009) 4206

[28] A.A. Chernov, Modern Crystallography III: Crystal Growth. Part I, Springer, 1984.

[29] M.B. Koss, J.C. LaCombe, L.A. Tennenhouse, et al., Metall. Mater. Trans. 30A (1999) 3179.

[30] V. Pines, A. Chait, M. Zlatkowski, J. Cryst. Growth 182 (1997) 219. 